\title{
Direct observation of effective ferromagnetic domains of cold atoms in a shaken optical lattice
}

\author{
Colin V. Parker ${ }^{1}$, Li-Chung $\mathrm{Ha}^{1}$ and Cheng Chin ${ }^{1,2 \star}$
}

\begin{abstract}
One of the intriguing properties of quantum many-body systems is the emergence of long-range order from particles with short-range interactions. For example, magnetism involves the long-range ordering of electron spins. Systems of ultracold atoms are rapidly emerging as precise and controllable simulators of magnetism and other phenomena. Spinor condensates $^{1,2}$ are a powerful tool in this regard; however, the spin interaction is typically weak and accessible only when multiple atomic internal states are collisionally stable. Here we demonstrate a lattice-shaking technique for hybridizing Bloch bands in optical lattices to introduce a strong effective spin interaction and the formation of large ferromagnetic domains. Our band hybridization method is independent of the atomic internal state, and can be widely applied to quantum simulators to explore new magnetic phases in optical lattices with tunable band structure.
\end{abstract}

In ultracold atomic systems, much effort has focused on reaching conditions where interparticle interactions are capable of generating long-range ordered phases. Efforts to simulate ferromagnetism using spinor Bose gases ${ }^{1-5}$ have taken advantage of contact and dipole interactions to form domains ${ }^{2}$ and spin textures $^{6-8}$. The resulting magnetic structure can be revealed by spin-dependent imaging techniques. However, the reliance on spindependent interactions ${ }^{9,10}$ leads to long characteristic timescales, so that equilibrium can be reached only under limited conditions ${ }^{11}$.

An alternative approach is to engineer stronger interactions by coupling atoms to a light field. For example, long-range order has been introduced using cavity photons to mediate interactions ${ }^{12}$, and by tilting an optical lattice ${ }^{13}$. With the recent development of more exotic lattices such as hexagonal ${ }^{14}$ and kagome ${ }^{15}$, as well as occupation of higher bands ${ }^{16}$, complicated band structures are possible where spin can be modelled using band properties rather than internal states. In this work we have developed a latticeshaking and imaging technique, which allows us to form large domains of cold atoms with strong ferromagnetic interactions and to fully map the domain structure. Complicated band structures can also be achieved by dynamically modulating the lattice ${ }^{17-20}$. Experiments using this technique have created band structures with negative or near-zero tunnelling coefficients ${ }^{18}$, or with multiple minima at high-symmetry points in the Brillouin zone ${ }^{19}$, which has allowed for the simulation of ferromagnetic systems ${ }^{21}$. Doublewell dispersions have also been obtained in the continuum by introducing Raman-dressed spin-orbit coupling ${ }^{22}$, and proposed to generate spatially ordered phases ${ }^{23,24}$.

Our experiment is based on a caesium Bose-Einstein condensate (BEC) of 25,000 atoms loaded into a one-dimensional optical lattice (Methods). Using lattice shaking at a frequency near the ground-band to first-excited-band transition, we create a hybridized band structure with two distinct energy minima at wavevectors $k= \pm k^{*}$, with $k^{*}$ incommensurate to the lattice (Fig. 1 and Supplementary Information). By adjusting the amplitude of the lattice shaking we can tune the dispersion from one with a single minimum to one with two distinct minima. We perform absorption imaging after time-of-flight (TOF) to determine the momentum distribution (see Fig. 1c for sample images). We also average over many shots to create a histogram, shown in Fig. 1d. For no shaking up to a shaking amplitude of about $15 \mathrm{~nm}$ we observe a single, narrow momentum distribution centred at zero, consistent with a regular BEC. As the shaking amplitude is increased further, we observe a bifurcation and the momentum distribution develops a two-peak structure. Compared to the calculated position of the minima from numerical diagonalization (white line in Fig. 1d, and see Supplementary Information), we find good overall agreement despite the fact that the calculations do not include interactions or the effect of the confining potential. In a spin analogy, with momentum playing the role of magnetization, the transition from one to two minima corresponds to a quantum analogue of the paramagnetic to ferromagnetic transition (see Supplementary Information).

In the ferromagnetic phase, most shots feature all of the atoms in one state or the other, that is, fully magnetized samples. This observation suggests that interactions are important and create a preference for occupation of a single minimum. At length scales greater than $\pi / k^{*}$ we can expand around the minima and treat the system as a two-mode BEC, with the minima labelled as spin-up and spin-down (see Fig. 1b). For a uniform system, this yields an effective Hamiltonian (Supplementary Information),

$$
H=\sum_{\sigma=\uparrow, \downarrow} \epsilon_{\sigma} N_{\sigma}+\frac{g}{2} N_{\uparrow}^{2}+\frac{g}{2} N_{\downarrow}^{2}+2 g N_{\uparrow} N_{\downarrow}
$$

where $\epsilon_{\sigma}$ represents the single-particle energy of each spin state, $N_{\uparrow}\left(N_{\downarrow}\right)$ is the number of up (down) spins, and $g=4 \pi \hbar^{2} a / m V$ is the interaction strength in terms of the scattering length $a$, the mass $m$, the effective trap volume $V$ and the Planck constant $h=2 \pi \hbar$. The factor of two in the interspecies interaction arises from inclusion of both Hartree (direct) and Fock (exchange) interactions. The exchange term, unique to systems with atoms in the same internal state, results in a ferromagnetic interaction for positive scattering length $a>0$. Introducing the collective spin $\mathbf{J}$ representation ${ }^{25}$, we find the Hamiltonian for an easy-axis magnet,

$$
H=\frac{\epsilon_{\uparrow}+\epsilon_{\downarrow}}{2} N+\frac{3 g}{4} N^{2}+\left(\epsilon_{\uparrow}-\epsilon_{\downarrow}\right) J_{z}-g J_{z}^{2}
$$

\footnotetext{
${ }^{1}$ The James Franck Institute and Department of Physics, University of Chicago, Chicago, Illinois 60637, USA, ${ }^{2}$ Enrico Fermi Institute, University of Chicago, Chicago, Illinois 60637, USA. *e-mail: cchin@uchicago.edu
} 


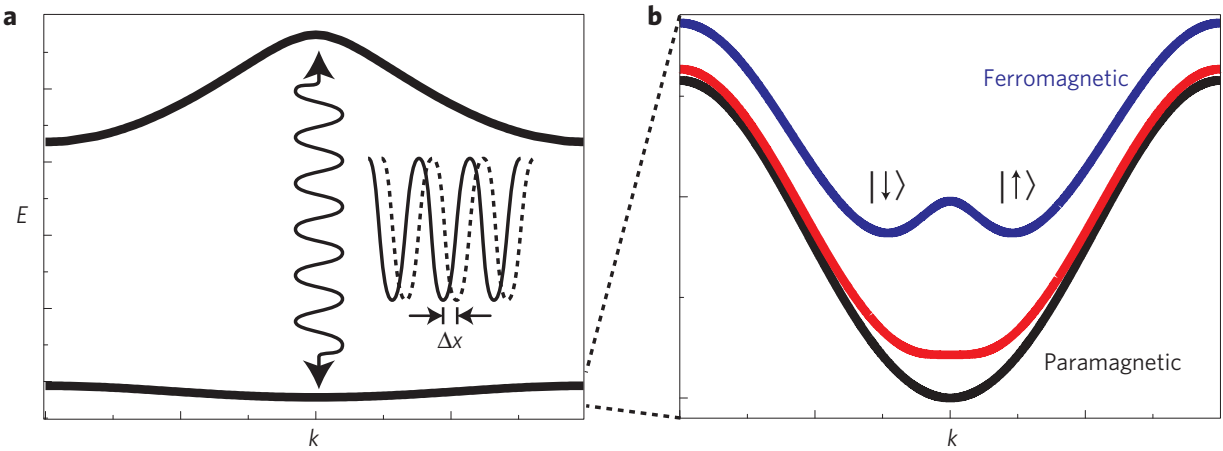

C
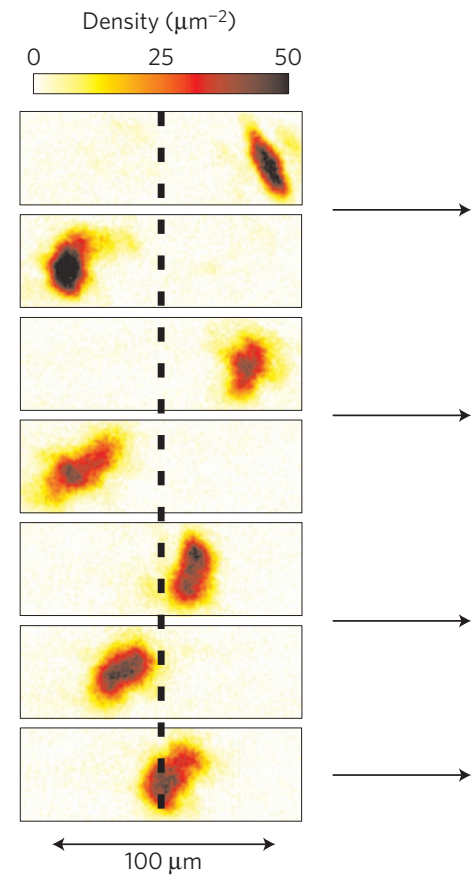

d
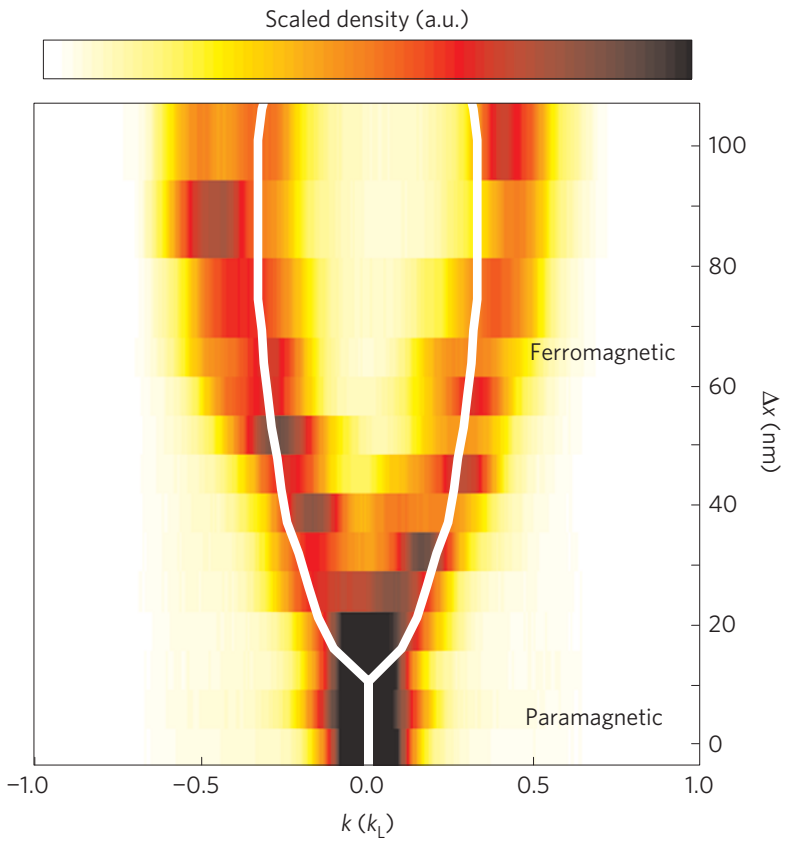

Figure 1 | Ferromagnetic transition in a shaken optical lattice with double-well dispersion. a, Dispersion $E(k)$ of the first two bands in an optical lattice, hybridized using near-resonant shaking. b. Expanded view of the hybridized ground band in the paramagnetic case with no shaking (black), the ferromagnetic case with strong shaking (blue), and the critical case (red). c, Single-shot images (at $30 \mathrm{~ms}$ TOF) of $\sim 25,000 \mathrm{Cs}$ atoms in the lattice with different shaking amplitudes. d, Momentum distribution along the lattice direction as a function of peak-to-peak shaking amplitude $\Delta x$, averaged over ten trials at each amplitude. Imbalances between the two minima are due to statistical uncertainties. The TOF position is used to determine the momentum in lattice units $k_{\mathrm{L}}=2 \pi / \lambda_{\mathrm{L}}$, where $\lambda_{\mathrm{L}} / 2=532 \mathrm{~nm}$ is the lattice constant. The white line is the calculated location of the dispersion minimum. We ramp up the shaking amplitude linearly over $50 \mathrm{~ms}$ followed by an additional $50 \mathrm{~ms}$ of constant shaking.

where $J_{z}=(1 / 2)\left(N_{\uparrow}-N_{\downarrow}\right)$ is the magnetization and $N=N_{\uparrow}+N_{\downarrow}$ is a constant of motion. Our ferromagnetic interaction derives from the large energy cost to maintain density waves, making it strong and comparable to the interaction between like spins.

We investigate the magnetization process by testing its sensitivity to an explicit energy imbalance $\left(\epsilon_{\uparrow}-\epsilon_{\downarrow}\right) J_{z}$ (ref. 21; see Fig. 2). This is realized by providing the condensate with a small initial velocity $v$ relative to the lattice that acts as a synthetic field $b=-v=\left(\epsilon_{\uparrow}-\epsilon_{\downarrow}\right) / 2 \hbar k^{*}$ (Supplementary Information). For a ferromagnet, the susceptibility will be infinite, and we expect to be able to fully magnetize the sample even for an energy imbalance $\epsilon_{\uparrow}-\epsilon_{\downarrow}$ much less than our temperature scale or chemical potential. To quantify the sensitivity we assume that atoms populate the two spin states according to a Boltzmann distribution with an effective temperature $T_{\text {eff }}$. When the lattice shaking is slowly ramped into the ferromagnetic regime over $100 \mathrm{~ms}$, we find a very sharp transition with an effective temperature of $0.7 \mathrm{nK}$, well below the actual temperature of $7 \mathrm{nK}$ and chemical potential $\sim 20 \mathrm{nK}$ determined from fitting the low-density tails of in situ profiles ${ }^{26}$. In terms of susceptibility, the transition is also 8 times sharper than what would be expected in the unshaken case. When ferromagnetism is ramped up more quickly, the sensitivity is reduced, which is the expected behaviour of a quenched ferromagnet. When the scattering length is reduced from 1.9 to $1.4 \mathrm{~nm}$ by a Feshbach resonance ${ }^{27}$, we observe a less sensitive transition, which confirms that spin interactions depend on the scattering length.

When a ferromagnet is cooled rapidly in the absence of an external bias field, domain formation is expected. Here we observe that with rapid ramping of the ferromagnetic interaction, domains are formed. When the shaking is weak, the barrier in the dispersion between the minima is non-existent or small, and the confining potential (which does not conserve quasimomentum) can move particles between the minima. Once the shaking amplitude is well above the critical value, the barrier forbids exchange of particles between the two minima, and the total 


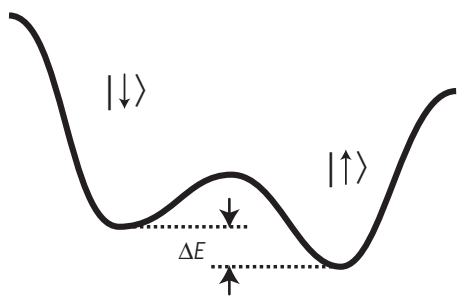

b

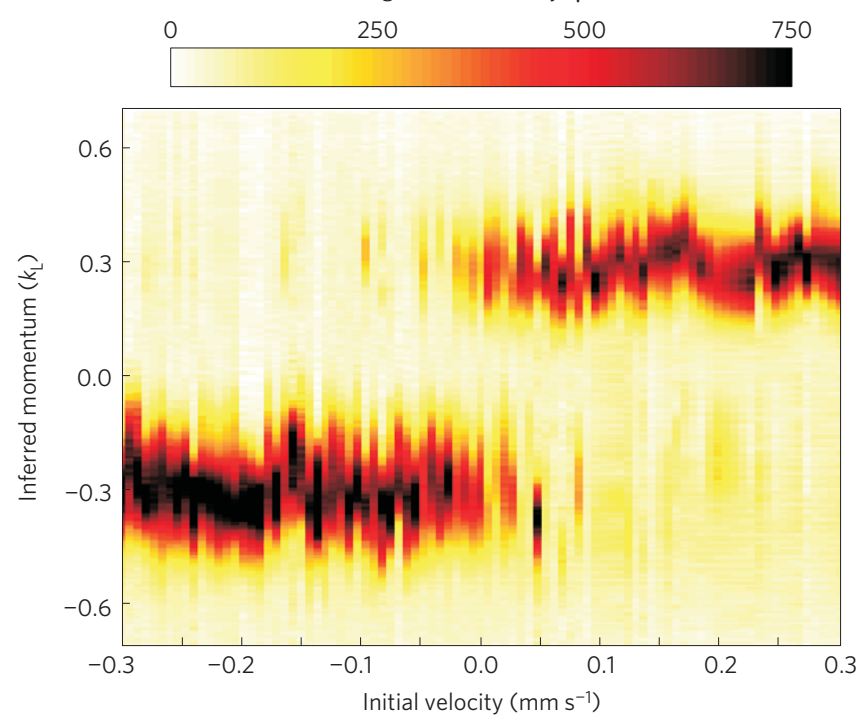

c

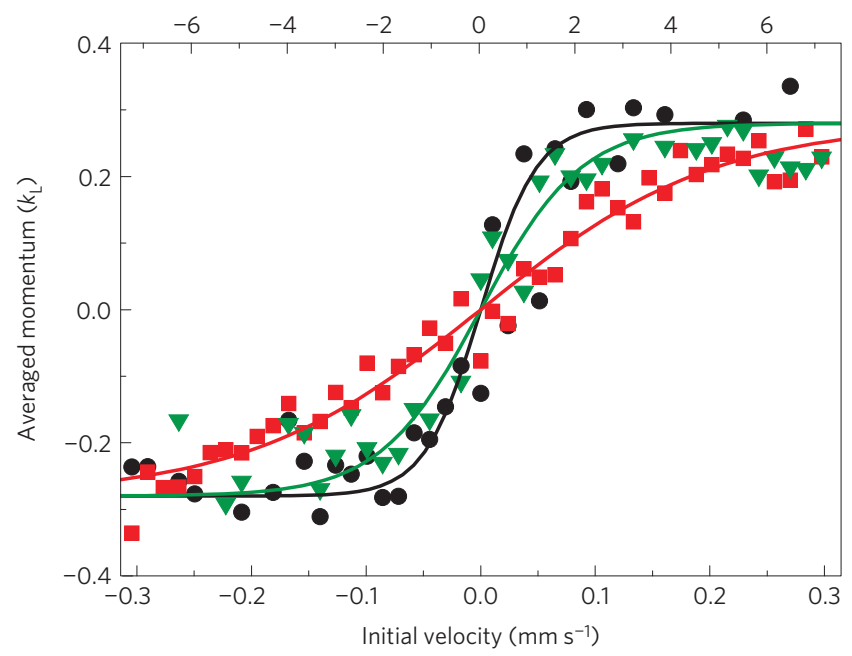

Figure 2 | Sensitivity of the ferromagnetic transition to explicit energy imbalance. a, We control the energy imbalance $\Delta E$ with a small initial velocity $v$ of the atoms relative to the lattice. For short times the imbalance is given by $\Delta E=2 \hbar k^{*} v$. $\mathbf{b}$, Average density profile along the lattice direction as a function of imbalance with $100 \mathrm{~ms}$ ramping time and scattering length $a=1.9 \mathrm{~nm}$. c, Average momentum as a function of imbalance under three different conditions: ramping time $100 \mathrm{~ms}$ with $a=1.9 \mathrm{~nm}$ (filled black circles) or $a=1.4 \mathrm{~nm}$ (green triangles), and ramping time $10 \mathrm{~ms}$ with $a=1.9 \mathrm{~nm}$ (red squares). The solid lines are fits to a thermal distribution, with effective temperatures $T_{\text {eff }}$ of 0.7 (black), 1.2 (green) and $2.9 \mathrm{nK}$ (red). The sample has temperature $T=7 \mathrm{nK}$ and chemical potential $\mu / k_{B} \approx 20 \mathrm{nK}$. Atoms were held at constant shaking amplitude $\Delta x=65 \mathrm{~nm}$ for $100 \mathrm{~ms}$ following the ramp. magnetization (that is, total quasi-momentum) will be conserved. Figure 3 shows typical single-domain and multi-domain samples. A detailed reconstruction of the original domain structure can be accomplished by taking advantage of the information in the Bragg peaks. Figure $3 \mathrm{~b}$ shows the relative weight between the main cloud and the lowest two Bragg peaks for single-domain samples with known magnetization. As the atoms are physically oscillating synchronously with the shaking, both wells possess the same averaged (physical) momentum at any point in the cycle (Supplementary Information). Therefore, the weights between the satellite Bragg peaks must be different to compensate for the $2 k^{*}$ difference in momentum of the central peak. Using these weights as a 'fingerprint', we can assign to any pixel a fraction of the density as spin-up density and the remainder as spin-down density. To do this we project the vector representing the relative Bragg weights onto the axis distinguishing spin-up from spin-down (Supplementary Information). Figure 3d shows a histogram of projected values. Using these values to identify the spin components allows a complete reconstruction of the original density and magnetization (Methods).

The reconstruction scheme reveals rich domain structures under different ramping paths (see Fig. 4). In the presence of domains, kinetic energy terms in all directions should be added to the Hamiltonian in equation (1) to describe the spatial pattern (Supplementary Information). When ferromagnetism is ramped up slowly over $100 \mathrm{~ms}$, we observe larger domains, with boundaries typically oriented in the direction of the short axis of the trap (Fig. 4a). When the ramping is done as a quench, over $10 \mathrm{~ms}$, we observe a greater number of smaller domains with less predictable orientation (Fig. 4b). Our result is consistent with the Kibble-Zurek mechanism in the sense that faster ramps yield shorter range correlations.

To quantify difference in domain size and shape, we compute the density-weighted magnetization correlator ${ }^{2}$,

$$
G(\delta \mathbf{r})=\frac{\left\langle\int j_{z}(\mathbf{r}) j_{z}(\mathbf{r}+\delta \mathbf{r}) \mathrm{d} \mathbf{r}\right\rangle}{\left\langle\int n(\mathbf{r}) n(\mathbf{r}+\delta \mathbf{r}) \mathrm{d} \mathbf{r}\right\rangle}
$$

where $n$ and $j_{z}$ denote number and magnetization densities, $\mathbf{r}$ and $\delta \mathbf{r}$ denote position coordinates, and the angle brackets denote an average over multiple trials. We distinguish between singleand multiple-domain samples (Supplementary Information). For fully polarized domains we expect $G(0)=1$; however, we obtain $G(0)=0.6$ for single-domain samples, which can be explained by our $\sim 16 \%$ fidelity for state identification. $G(0)$ is even lower for multiple-domain samples owing to the observed domain wall size, which is limited by atom dynamics during the $5 \mathrm{~ms}$ TOF. Along the short trap axis, the correlations in samples with slow ramping are both stronger (indicating fewer domain boundaries) and longer range (indicating larger domains) compared with quenched samples. In quenched samples the correlations are roughly isotropic owing to the random orientation of domains. In samples with slow ramping and multiple domains, the correlation along the long trap axis drops off abruptly at about $10 \mu \mathrm{m}$ or 20 lattice spacings (see Fig. 4d). Our analysis of $G(r)$ demonstrates that long-range spin correlations can be established, and that domain boundaries prefer to align along the short trap axis when ferromagnetism is turned on slowly. The effective mass along the shaking direction with $65 \mathrm{~nm}$ shaking is only 1.1 times the bare mass, making the system nearly isotropic. Therefore, the domain orientation is more likely related to minimizing the domain boundary area, and suggests that the system is in a low-temperature state. We note that even a rapidly quenched domain structure can quickly relax by exchanging energy with other degrees of freedom, for example, kinetic energy in the non-lattice directions (Supplementary Information). 

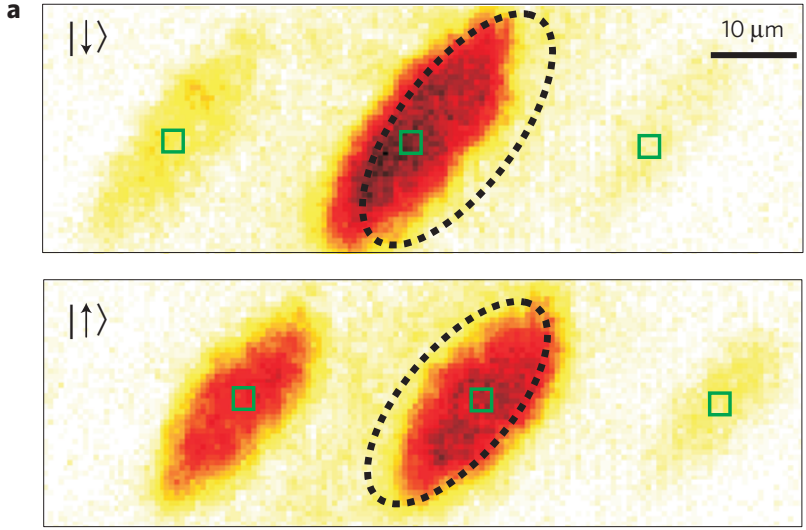

c
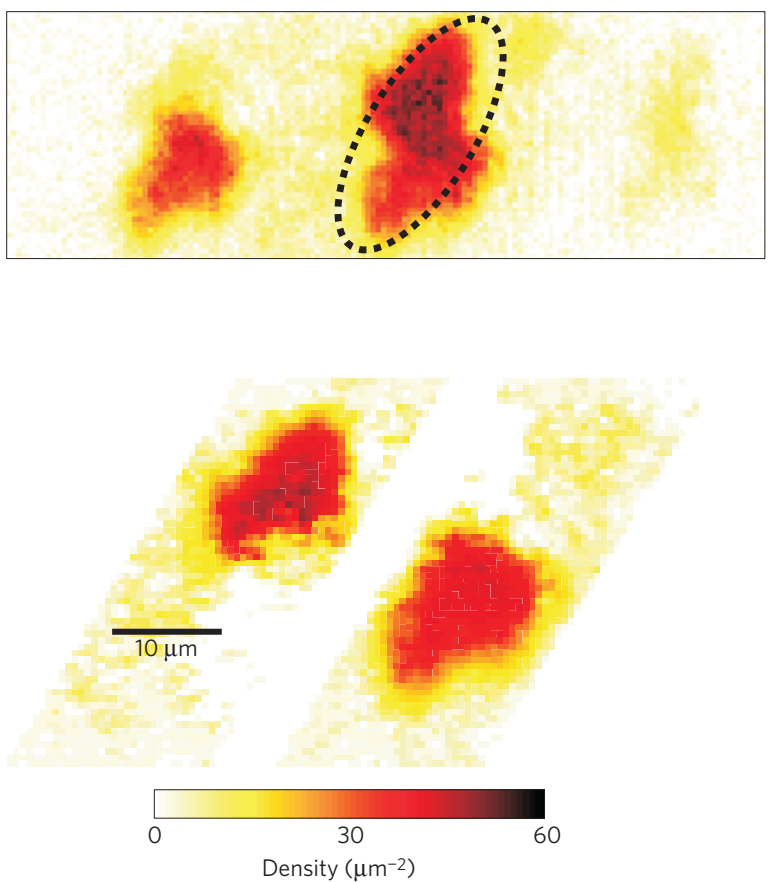

b

$\omega_{\downarrow}$

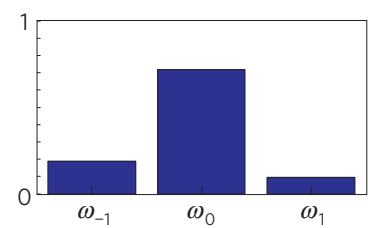

$\omega \uparrow$

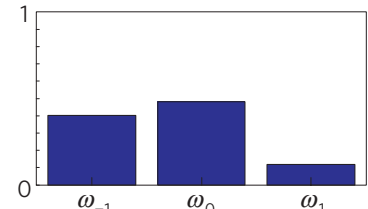

d
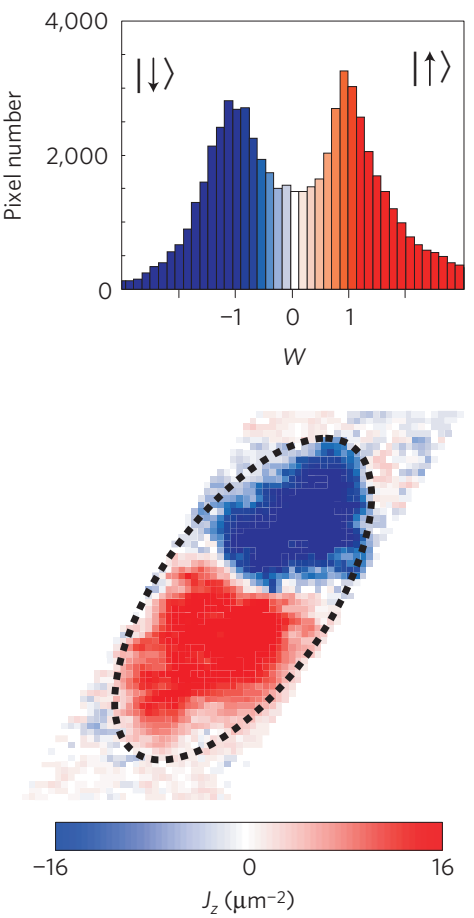

Figure 3 | Domain reconstruction. a, Images at $5 \mathrm{~ms}$ TOF for two oppositely oriented single-domain samples. b, Vectors $\boldsymbol{\omega}_{=}\left(\omega_{-1}, \omega_{0}, \omega_{1}\right)$ signifying relative weights between the -1st-, Oth- and 1st-order Bragg peaks, corresponding to the regions in a identified by squares. c, Image at $5 \mathrm{~ms}$ TOF for a two-domain sample. $\mathbf{d}$, Histogram (over 19 images) of pixel-wise projection onto $\boldsymbol{\omega}_{\uparrow}-\boldsymbol{\omega}_{\downarrow}$, denoted $\boldsymbol{W}$. The two peaks indicate that most of the atoms are either spin-up or spin-down. e, The extracted density of spin-down (left) and spin-up (right) atoms. f, The extracted density distribution of magnetization $j_{z}$. The dotted ovals in $\mathbf{a}, \mathbf{c}$ and $\mathbf{f}$ show the position of the samples before TOF. We ramp up the shaking amplitude over $100 \mathrm{~ms}$ followed by an additional $100 \mathrm{~ms}$ of constant shaking. The scattering length is $1.9 \mathrm{~nm}$.

Using lattice shaking to tune band structure has important implications for the simulation of various ordered states in solid systems, where Fermi surface shape and topology can play a very important role. The same double well used here would have a nested Fermi surface and be expected to undergo a charge density wave transition ${ }^{28}$, for example. Furthermore, the nearresonant shaking technique is easily extendable to two or three dimensions, or other atomic species, which can be fermionic and/or contain multiple accessible internal states. Thus, near-resonant shaking opens the door to a variety of exciting possibilities for quantum simulation.

\section{Methods}

Lattice loading. Our experiment begins by evaporating and loading a ${ }^{133} \mathrm{Cs} \mathrm{BEC}$ into a three-dimensional optical dipole trap with trapping frequencies of 8.6, 19.1 and $66.9 \mathrm{~Hz}$ in three directions, with the tightest trapping in the direction of gravity and imaging ${ }^{29}$. The atoms are then loaded into a one-dimensional optical lattice at $35^{\circ}$ to the in-plane trapping directions, where the final atom number is between 20,000 and 30,000 at a temperature of $7 \mathrm{nK}$. Our optical lattice is formed by reflecting one of the dipole trap beams back on itself after passing through two oppositely oriented acousto-optic modulators (AOMs). For our experiments we use a laser wavelength $\lambda_{\mathrm{L}}=1,064 \mathrm{~nm}$ (lattice spacing $532 \mathrm{~nm}$ ) and lattice depth $V=7.0 E_{\mathrm{R}}$, where $E_{\mathrm{R}}=h^{2} / 2 m \lambda_{\mathrm{L}}^{2}$ is the lattice recoil energy.

Lattice shaking. After the atoms are loaded into the optical lattice, a sinusoidal shaking is turned on with a linear ramp of between 5 and $100 \mathrm{~ms}$. After the shaking is ramped up, we shake the atoms for $50-100 \mathrm{~ms}$ before performing an in situ image or extinguishing all lattice and trapping light for a TOF image. When the lattice is removed abruptly for TOF, the atoms in different spin states are projected back to plane waves with different momenta, giving us an effective Stern-Gerlach measurement. Moderate heating is observed during the lattice shaking, resulting in evaporation that leads to a $1 / e$ lifetime of $1 \mathrm{~s}$ for the particle number. Nonetheless, $\mathrm{BEC}$ can be maintained for $>1 \mathrm{~s}$.

The lattice modulation is accomplished by frequency modulating the driving radiofrequency (around a carrier of $80 \mathrm{MHz}$ ) for the paired AOMs, which changes 


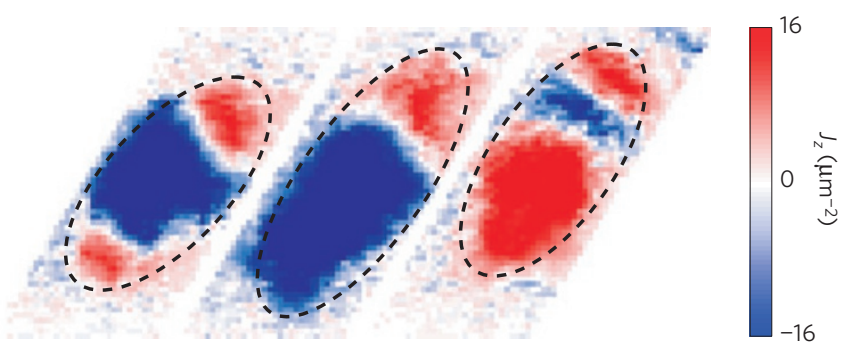

b

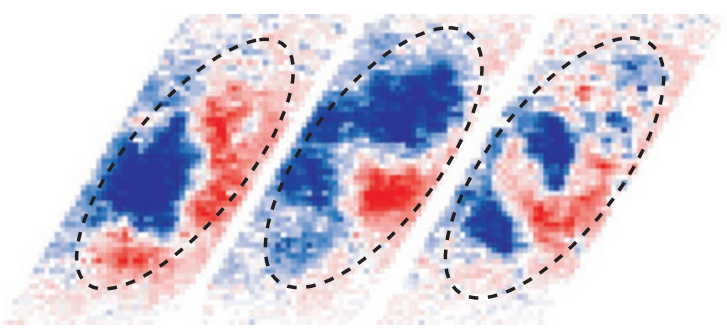

d

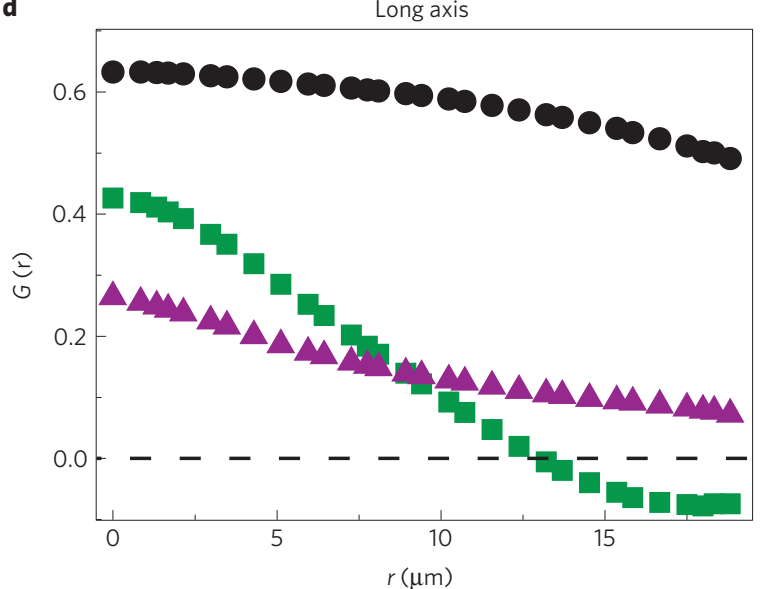

c
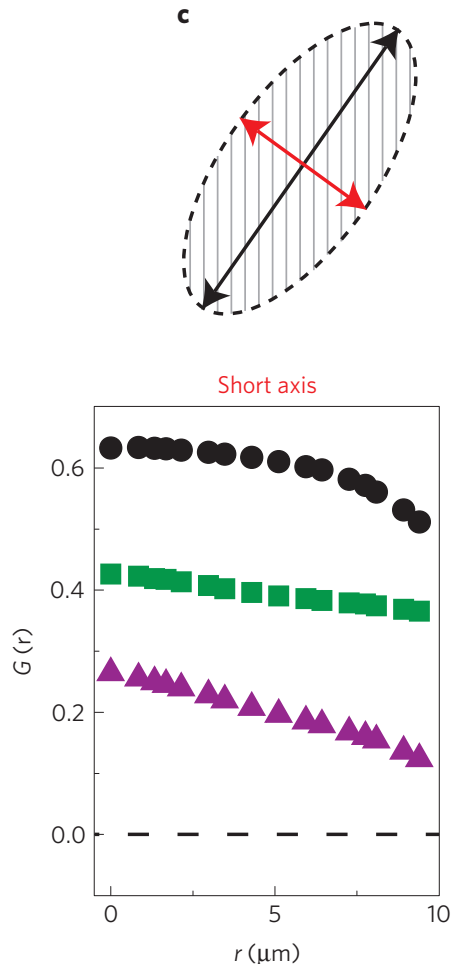

Figure 4 | Ferromagnetic domains and spin correlations. a, Three representative magnetization images with 100 ms ramping time. b, Three representative magnetization images with 10 ms ramping time. c, Illustration showing trap geometry and axes. d, Density-weighted magnetization correlator $G(r)$ along the long and short trapping directions: the average of 12 single-domain samples with $100 \mathrm{~ms}$ ramping time (filled black circles), 11 multi-domain samples with $100 \mathrm{~ms}$ ramping time (green squares), and 19 multi-domain samples with $10 \mathrm{~ms}$ ramping time (purple triangles). Atoms were held at constant shaking amplitude $\Delta x=65 \mathrm{~nm}$ for $100 \mathrm{~ms}$ following the ramp. The scattering length was $1.9 \mathrm{~nm}$.

the relative phase, and therefore the optical path length, between the AOMs (Supplementary Information). To realize a double-well dispersion, we use a sinusoidal shaking of the optical lattice at a frequency near the ground-band to first-excited-band transition at zero quasi-momentum. This shaking allows the two bands to mix, creating a competition between the positive curvature of the ground band and the negative curvature of the excited band, as shown in Fig. 1. Our zero-momentum bandgap is $5.0 E_{\mathrm{R}}$, with an effective mass 2.9 times the bare mass. We apply the shaking at a slightly blue-detuned frequency of $7.3 \mathrm{kHz}=5.5 E_{\mathrm{R}} / \mathrm{h}$, which gives the least heating when the double-well dispersion is formed. Shaking near the transition allows us to obtain significant hybridization with only a small shaking amplitude, reducing the heating. The solid black curves in Fig. 1b show the lowest two bands without shaking in the dressed atom picture. To confirm that the bands will mix to create a double-well potential, we have numerically computed the hybridized Floquet states for several different shaking amplitudes and the results agree well with the experiment (Supplementary Information).

Domain reconstruction. The two peaks of the histogram in Fig. $3 \mathrm{~d}$ are used to define pure spin-up or spin-down, and intermediate values indicate the presence of both spin components. With the spin components isolated, we can adjust for the physical displacement accumulated during the $5 \mathrm{~ms}$ TOF due to the momentum $\pm k^{*}$ and determine the original density and spin distributions (see Fig. 3e,f). Our imaging is limited to density and magnetization projections along the imaging axis; however, the sample is much thinner perpendicular to the imaging axis $(\sim 4 \mu \mathrm{m})$, and we expect few domain walls perpendicular to the imaging direction.
Received 9 August 2013; accepted 18 September 2013; published online 20 October 2013

\section{References}

1. Stenger, J. et al. Spin domains in ground-state Bose-Einstein condensates. Nature 396, 345-348 (1998).

2. Sadler, L. E., Higbie, J. M., Leslie, S. R., Vengalattore, M. \& Stamper-Kurn, D. M. Spontaneous symmetry breaking in a quenched ferromagnetic spinor Bose-Einstein condensate. Nature 443, 312-315 (2006).

3. Schmaljohann, H. et al. Dynamics of $F=2$ spinor Bose-Einstein condensates. Phys. Rev. Lett. 92, 040402 (2004).

4. Chang, M-S., Qin, Q., Zhang, W., You, L. \& Chapman, M. S. Coherent spinor dynamics in a spin-1 Bose condensate. Nature Phys. 1, 111-116 (2005).

5. Stamper-Kurn, D. M. \& Ueda, M. Spinor Bose gases: Symmetries, magnetism, and quantum dynamics. Rev. Mod. Phys. 85, 1191-1244 (2013).

6. Vengalattore, M., Leslie, S. R., Guzman, J. \& Stamper-Kurn, D. M. Spontaneously modulated spin textures in a dipolar spinor Bose-Einstein condensate. Phys. Rev. Lett. 100, 170403 (2008).

7. Kronjäger, J., Becker, C., Soltan-Panahi, P., Bongs, K. \& Sengstock, K. Spontaneous pattern formation in an antiferromagnetic quantum gas. Phys. Rev. Lett. 105, 090402 (2010).

8. Vinit, A., Bookjans, E. M., de Melo, C. A. R. S. \& Raman, C. Antiferromagnetic spatial ordering in a quenched one-dimensional spinor gas. Phys. Rev. Lett. 110, 165301 (2013). 
9. Ho, T-L. Spinor Bose condensates in optical traps. Phys. Rev. Lett. 81, 742-745 (1998).

10. Ohmi, T. \& Machida, K. Bose-Einstein condensation with internal degrees of freedom in alkali atom gases. J. Phys. Soc. Jpn 67, 1822-1825 (1998).

11. Guzman, J. et al. Long-time-scale dynamics of spin textures in a degenerate $F=1{ }^{87} \mathrm{Rb}$ spinor Bose gas. Phys. Rev. A 84, 063625 (2011)

12. Baumann, K., Guerlin, C., Brennecke, F. \& Esslinger, T. Dicke quantum phase transition with a superfluid gas in an optical cavity. Nature 464, 1301-1306 (2010).

13. Simon, J. et al. Quantum simulation of antiferromagnetic spin chains in an optical lattice. Nature 472, 307-312 (2011).

14. Soltan-Panahi, P. et al. Multi-component quantum gases in spin-dependent hexagonal lattices. Nature Phys. 7, 434-440 (2011).

15. Jo, G-B. et al. Ultracold atoms in a tunable optical kagome lattice. Phys. Rev. Lett. 108, 045305 (2012).

16. Wirth, G., Ölschläger, M. \& Hemmerich, A. Evidence for orbital superfluidity in the $p$-band of a bipartite optical square lattice. Nature Phys. 7, 147-153 (2011).

17. Gemelke, N., Sarajlic, E., Bidel, Y., Hong, S. \& Chu, S. Parametric amplification of matter waves in periodically translated optical lattices. Phys. Rev. Lett. 95, 170404 (2005)

18. Lignier, H. et al. Dynamical control of matter-wave tunneling in periodic potentials. Phys. Rev. Lett. 99, 220403 (2007).

19. Struck, J. et al. Quantum simulation of frustrated classical magnetism in triangular optical lattices. Science 333, 996 (2011).

20. Struck, J. et al. Tunable gauge potential for neutral and spinless particles in driven optical lattices. Phys. Rev. Lett. 108, 225304 (2012).

21. Struck, J. et al. Engineering Ising-xy spin-models in a triangular lattice using tunable artificial gauge fields. Nature Phys. http://dx.doi.org/10.1038/nphys2750 (2013).

22. Lin, Y-J., Jiménez-García, K. \& Spielman, I. B. Spin-orbit-coupled Bose-Einstein condensates. Nature 471, 83-86 (2011).

23. Wang, C., Gao, C., Jian, C-M. \& Zhai, H. Spin-orbit coupled spinor Bose-Einstein condensates. Phys. Rev. Lett. 105, 160403 (2010).
24. Ho, T-L. \& Zhang, S. Bose-Einstein condensates with spin-orbit interaction. Phys. Rev. Lett. 107, 150403 (2011).

25. Dalton, B. J. \& Ghanbari, S. Two mode theory of Bose-Einstein condensates: interferometry and the Josephson model. J. Mod. Opt. 59, 287-353 (2011).

26. Zhang, X., Hung, C-L., Tung, S-K. \& Chin, C. Observation of quantum criticality with ultracold atoms in optical lattices. Science 335, 1070-1072 (2012).

27. Chin, C., Grimm, R., Julienne, P. \& Tiesinga, E. Feshbach resonances in ultracold gases. Rev. Mod. Phys. 82, 1225-1286 (2010).

28. Shankar, R. Renormalization-group approach to interacting fermions. Rev. Mod. Phys. 66, 129-192 (1994).

29. Hung, C-L., Zhang, X., Gemelke, N. \& Chin, C. Accelerating evaporative cooling of atoms into Bose-Einstein condensation in optical traps. Phys. Rev. A 78, 011604 (2008).

\section{Acknowledgements}

We thank C-K. Lin for assistance in the early stages of the experiment. We acknowledge useful discussions with N. Gemelke, I. Spielman, A. Rançon, H. Zhai and G. Baym. This work was supported by NSF MRSEC (DMR-0820054), NSF Grant No. PHY-0747907 and ARO Grant No. W911NF0710576 with funds from the DARPA OLE Program.

\section{Author contributions}

L-C.H. performed the experiments. L-C.H. and C.V.P. analysed the data and C.V.P. wrote the manuscript. C.C. supervised.

\section{Additional information}

Supplementary information is available in the online version of the paper. Reprints and permissions information is available online at www.nature.com/reprints. Correspondence and requests for materials should be addressed to C.C.

\section{Competing financial interests}

The authors declare no competing financial interests. 